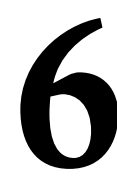

\title{
ADOLESCENCIA, TIEMPO LIBRE Y EDUCACIÓN. UN ESTUDIO CON ALUMNOS DE LA ESO
}

\author{
(ADOLESCENCE, FREE TIME AND EDUCATION. A STUDY WITH STUDENTS \\ FROM E.S.O. COMPULSORY SECONDARY EDUCATION)
}

José Manuel Muñoz Rodríguez

y Susana Olmos Migueláñez

Universidad de Salamanca

\section{RESUMEN}

Presentamos el resultado de una investigación cuyo objetivo ha sido analizar la distribución, usos y valores que sostienen el tiempo libre de los estudiantes de Educación Secundaria Obligatoria (ESO) de Salamanca.

El instrumento de recogida de datos ha sido la encuesta; el muestreo aleatorio simple de unidades por conglomerados, dispuesto por centros escolares y estratos, según localización geográfica (rural, urbana) y pertenencia al sector público o privado. El análisis se efectuó a partir de tres variables: el nivel de estudios $\left(1^{\circ}, 2^{\circ}, 3^{\circ}\right.$ y $\left.4^{\circ} \mathrm{ESO}\right)$, el género, y la ubicación geográfica, si bien el trabajo se centra en la primera de ellas. Los resultados muestran escasez de valores educativos en su tiempo libre y diferencias significativas a tener en cuenta en función del nivel educativo. El tiempo libre es un tiempo crucial en la vida de los adolescentes y presenta procesos análogos a los experimentados en el tiempo escolar.

\section{ABSTRACT}

We offer the results of a piece of research that had as aim to analyse the distribution and use of the free time among the E.S.O. students in Salamanca, as well as the values that support it.

The data has been collected by means of a survey and by establishing a simple random sampling of units in conglomeration, arranged in educational 
establishments and strata, according to their geographical location (rural, urban) and their belonging to the public or private sector. The analysis was carried out based on three variables: the course level or level of studies (1st, $2 \mathrm{nd}$, 3rd, 4th E.S.O.), the student gender, and the geographical location, although this research has focuses on the first of them. The results show a lack of educational values in the adolescent free time and significant differences to be taken into account depending on their educational level. The spare time adolescents enjoy represents a key time in their lives and exhibits analogous processes to those experimented during their time spent at school.

\section{PLANTEAMIENTO DE LA CUESTIÓN}

Nadie pone en duda la importancia del tiempo para la vida de las personas. Nos guste o no, somos seres temporales. La vida misma es una síntesis entre la persona y el tiempo, cuya unión intrínseca no nos permite separarlos más que en la teoría, afectando a sus modos de vida, a sus relaciones y comunicaciones, a su existencia y, en consecuencia, a su educación. Todo cuanto somos y hacemos se encuentra inmerso en un cruce de pertenencias y tramas temporales, a la vez que espaciales, que nos obliga a no dejar de mirar y analizar el tiempo, pues en él encontramos indicios del buen hacer del ser humano y las claves para conseguirlo (Zubiri, 1996).

Actualmente vivimos con la sensación de faltarnos tiempo. Una carencia, fruto de los ritmos vitales y de la multiplicidad de los tiempos propios de la sociedad contemporánea, que nos lleva a pensar, como exponente del planteamiento del tema, que la calidad de vida de las personas y, en consecuencia, sus actividades y relaciones sociales, la construcción de su identidad individual y colectiva, su educación en definitiva, mejorarían si se conociesen y se coordinasen de forma más óptima los tiempos sociales. En este sentido, si el tiempo es vital para las personas, de forma análoga resulta vital su conocimiento, distribución y uso, si de procesos de construcción de la persona, procesos educativos, estamos hablando (Gimeno, 2008).

La riqueza y variabilidad conceptual con la que se aborda el tiempo nos permite hablar de diversas formas del tiempo (McNamara, 1997) entre las que destaca el tiempo libre. Es una realidad ambivalente y contradictoria, pues podemos llenarlo de aquellas pequeñas cosas que terminan marcando positivamente nuestra vida o, más bien, de pequeños detalles y actitudes que dejan mucho que desear. El tiempo libre es un tiempo, de entrada, liberado de ocupaciones que puedes acomodar a tu gusto y manera. Es una parte del tiempo vital de una persona, de libre disposición, para llevar a cabo cuantas actividades se consideren oportunas. "El tiempo libre debe entenderse no como estar libre de algo, sino también como estar libre para algo, o sea, dis- 
ponibilidad para ejecutar algo que procura placer y satisfacción, etc." (Lobo y Menchén, 2004, 44-45).

Y si para alguien tiene especial sentido y pertinencia este tiempo libre es para los jóvenes, principalmente para ese grupo de jóvenes que están despertando a la autonomía en relación a ese tiempo: los adolescentes (Comas, 2000; Saz-Marín, 2007). Las nuevas oportunidades de diversión y entretenimiento de la sociedad de la información y la comunicación, —-sociedad del ocio y del consumo-, abre nuevos cauces al desarrollo personal de los adolescentes que de ser explícitos y analizados pueden suponer una experiencia humana que ayude a mejorar la calidad de vida y a manifestar nuevas aptitudes, valores, conocimientos y habilidades; en definitiva, a mejorar en todo lo que afecta al proceso de socialización y de desarrollo comunitario.

De entre los muchos grupos de adolescentes que podemos encontrarnos, existe un grupo, fácilmente identificable: los alumnos de Educación Secundaria Obligatoria. Siendo conscientes de que se trata de un grupo muy heterogéneo, es precisamente dicha particularidad la que hace atractivo al grupo, pues a pesar de ella, los chicos conviven conjuntamente en el entorno escolar. Y siendo así, en cierta medida, pensamos que muchos de los fenómenos y procesos bajo los que conviven en el centro de forma conjunta terminan encontrando prolongación, llevan anexos, fenómenos y procesos educativos en el denominado tiempo libre. Es decir, si por imperativo estructural dichos alumnos tan dispares deben convivir en el centro escolar, no podemos obviar que como grupo tiene su importancia y, por tanto, resulta pertinente acercarnos a análisis conjuntos también fuera del entorno escolar, sin pretender establecer relaciones lineales y mecánicas entre tiempo dentro de la escuela y los tiempos libres vividos más allá de los muros.

Y en el centro de todo ello la educación en cuanto que proceso vitalmente necesario, convirtiendo el tiempo libre en tiempo potencialmente educativo y la educación en el tiempo libre como aquella educación que trabaja en un tiempo que va más allá del tiempo planificado, programado, establecido para ser evaluado progresivamente en aras de conseguir la buena educación y que, por el contrario, se centra en un concepto de educación marcado por la posibilidad de ejercer la dimensión ética de lo humano (Morín, 2006). Una educación que reafirma la inquietud del ser, marcada al compás de los valores cuya variable temporal, de ser analizada, nos proporciona unas claves para que los adolescentes entiendan que el tiempo libre puede llegar a ser comprendido como fuente de felicidad, satisfacción, autodesarrollo y autorrealización, más allá del conformismo y del tiempo libre vivido como ocio superficial y consumista. 


\section{CONTEXTUALIZACIÓN Y FUNDAMENTACIÓN DE LA INVESTIGACIÓN}

Son numerosas las investigaciones que sobre juventud y tiempo libre se han llevado a cabo ${ }^{1}$. Menos son aquellas que inciden exclusivamente en los alumnos de la etapa de enseñanza secundaria fuera del entorno escolar. Aún así, coincidimos en que es ahí, en los conflictos que surgen en el seno de una misma etapa educativa, donde hemos de buscar aspectos que pueden verse optimizados tanto desde dentro como desde fuera del contexto escolar.

El tema que nos ocupa, tras ostentar un lugar poco relevante en el imaginario social e institucional, se encuentra actualmente convertido en un tema importante para nuestra sociedad en general, y para Castilla y León en particular, pues se centra en un sector emergente que deja entrever posibilidades de desarrollo ${ }^{2}$. De hecho, en este comienzo de siglo, una parte sustancial de las políticas de juventud ha girado en torno al tema del ocio, del tiempo libre y de los fines de semana. No obstante, pensamos que la preocupación surgida en estos últimos años en torno a sectores -minorías interculturales, adultos, tercera edad, mujeres-, han dejado de lado al colectivo adolescente.

Si bien vamos a presentar los resultados de una investigación sobre la distribución, usos y valores que sostiene el tiempo libre de los alumnos de la ESO salmantinos, 12-16 años, desde un punto de vista educativo y en función del nivel educativo, estos resultados se insertan en un estudio que ha tenido una pretensión mayor, en base a una triple perspectiva: en función del género, en función de la localidad, rural-urbano, y en función del nivel educativo $^{3}$, cuyo objetivo general ha sido el de presentar unos parámetros que ayuden a la optimización del tiempo libre de los jóvenes-adolescentes, a una mejora en su distribución, organización y aprovechamiento de los tiempos de su vida, ayudando a reorganizar todas aquellas actividades llevadas a cabo por ellos y que suponen un consumo innecesario de tiempo libre, incluso cuando portan el rótulo de educativas.

En definitiva estudiamos los usos y la distribución del tiempo libre en los jóvenes-adolescentes de Salamanca y provincia para comprobar, por un lado, si podemos hablar de un tiempo marcado por el ocio o, más bien, de un tiempo estéril, y pasivo; y, por otro lado, si las actividades llamadas educativas, llevadas a cabo por dicho colectivo, son en sí formativas o, por el contrario, estamos hablando de motivos que tienen que ver más con el ocio como objeto de consumo. Si el tiempo libre termina siendo punto fundamental en la construcción de la identidad personal y colectiva, hemos de incidir en el análisis de los usos y la distribución de dicho tiempo para com- 
probar los valores y los espacios, las actividades y las relaciones que lo sustentan (Fernández y Carmona, 2009).

La hipótesis que formulamos es la siguiente: si el tiempo es una de las dimensiones fundamentales de la persona, y el tiempo libre termina siendo uno de los elementos definitorios de su personalidad, cabe la posibilidad de pensar que si hacemos reflexionar a los adolescentes sobre los usos y la distribución de su tiempo libre obtendrán unos referentes que les ayudarán a pensar que existen otras maneras de vivirlo y, más aún, a reflexionar sobre las posibles formas como les gustaría llegar a vivirlo, pues en ocasiones muchas de las llamadas actividades educativas terminan por no tener tal condición.

Una cuestión que, de entrada, tiene dos vertientes fundamentales que la contextualizan: una sociológica, puesto que las condiciones de vida han cambiado y el ritmo y distribución de los tiempos están, hoy día, sujetos a otro tipo de condicionantes distintos de los tradicionales; y otra pedagógica, ya que la aceleración en los ritmos vitales actuales y la indispensable necesidad de información y formación requieren, quizá, de nuevos espacios, modelos e instituciones que ofrezcan alternativas a los problemas actuales. La clave, pues, del estado actual del tema se centra en la búsqueda de procesos a partir de los cuales los jóvenes lleguen a conocerse a sí mismos en base a las interrelaciones que mantienen con su tiempo en general y con su tiempo libre en particular, en relación con su estilo de vida y con sus inquietudes de futuro.

Somos conscientes del vasto campo en el que nos enmarañamos; más aún, que estudiar el tiempo libre desde un punto de vista educativo nos obligaría a estudiar dicha variable desde múltiples significados y diferentes problemáticas (Muntañola, 2007), entre otros aspectos, porque no todo tiempo libre es a su vez tiempo educativo, ni todo tiempo rotulado de formativo es educativo y sirve para la construcción de la persona, por señalar algunos. Pero la inquietud que despiertan algunos hechos actuales - pérdida de autoridad por parte de padres y maestros, excesivo uso de las nuevas tecnologías en el tiempo libre, pérdida de valores tradicionales y del sentido vital del tiempo, entre otras- tiene más valor que los problemas que emergen. El tiempo en relación con la persona es lineal e irreversible pero, también, es probable y posible, en cuya conformación la educación es clave (Romero, 2000).

Esta centralidad educativa nos permite, a su vez, presentar una perspectiva educativa del tiempo libre en cuanto que terreno óptimo para el desarrollo de la persona, ámbito donde se desarrollan valores, relaciones y comunicaciones, en cuyo encuentro tenemos la fuente necesaria para el de- 
sarrollo de identidades personales y colectivas, elemento central de cualquier proceso educativo. Vivir el tiempo libre de forma óptima es un derecho educable (Caride, 2007) y, en consecuencia, la cultura y, sobre todo, la educación, deben reivindicar su papel protagonista en la generación de capacidades y oportunidades para vivir y degustar el tiempo libre en armonía con los intereses y necesidades vitales de cada colectivo de personas, en base a criterios no sólo económicos y organizativos sino, principalmente, sociales y educativos.

Desde esta base, siguiendo a Zuzanek (1980; citado en Caride 1998), caben cuatro enfoques conceptuales del tiempo libre desde los que, a nuestro juicio, poder plantear cualquier perspectiva educativa del tiempo libre; cuatro perspectivas donde la educación encuentra un sentido en el vasto campo en el que estamos:

- La primera hunde sus raíces en la concepción del tiempo libre como factor básico para el desarrollo integral de la persona, objetivo básico de cualquier proceso primario de formación.

- La segunda justifica la educación en cuanto que elemento que garantiza el derecho a un tiempo libre positivo y, por tanto, previene de usos nocivos del mismo.

- La tercera inserta la educación en el mundo de la vida de las personas, en sus acciones y relaciones cotidianas, siendo el tiempo libre uno de los elementos integrantes de este mundo particular de cada uno tanto desde una dimensión individual como comunitaria.

- La cuarta sitúa la educación como ámbito con entidad propia para desarrollar actividades formativas en el tiempo libre.

Asumimos, pues, una perspectiva educativa del tiempo libre que aglutina y conjuga tanto los planteamientos que tienen que ver con una educación en el tiempo libre -el tiempo libre como ámbito donde desarrollar acciones educativas-, como aquellos que inclinan su interés en educar para el tiempo libre - el tiempo libre en cuanto que móvil de la intervención pedagógica-. Más aún, de igual forma pensamos que la propuesta híbrida que en su momento hicieron Puig y Trilla (1987) y que hoy se mantiene vigente, educar mediante el tiempo libre, es válida y pertinente. Con ella justificamos investigaciones como las que presentamos, pues nada mejor que educar a los chicos mediante investigaciones que reflejan las actividades que realizan ellos mismos y los valores que las sostienen para hacerles ver, desde su propia actividad y actitud, cómo es el empleo de su tiempo libre y cómo podría llegar a ser. 
La educación, dentro de este terreno, tiene como principal objetivo no tanto el plantear por plantear actividades dentro del tiempo libre sino que, más bien, dichas actividades que desarrollan habitualmente en su tiempo libre encuentren significación y sentido para su desarrollo personal y comunitario, por un lado, y, por otro lado, que las actividades que se planteen para realizar en el tiempo libre contengan un valor formativo, de desarrollo social y de transformación social. Es el modo de conseguir que la educación haga del tiempo libre un tiempo de creatividad, satisfacción, disfrute, en definitiva, un área específica de desarrollo personal y comunitario de la especie humana.

"El objeto general de la educación del tiempo libre es ayudar a... que alcancen una calidad de vida anhelado mediante el tiempo libre. Esto se puede lograr mediante el desarrollo y estímulo de valores, actitudes, conocimientos y destrezas del tiempo libre, mediante el desarrollo personal, social, físico, afectivo e intelectual. Esto, a su vez, tendrá un impacto sobre la familia, la comunidad y la sociedad toda" (WLRA, 1993).

En suma, defendemos el binomio educación-tiempo libre al objeto de desarrollar procesos educativos a lo largo y ancho de toda la vida, en cualquier espacio y tiempo, en base a diferentes recursos y educadores, de estimular la creatividad y el espíritu crítico mediante la transmisión de valores y capacidades para un correcta socialización y participación social, de hacer visible y real la autonomía y libertad del individuo en sus acciones y prácticas sociales. Asumimos, pues, una referencia a la educación en el tiempo libre en estrecha conexión con conceptos como el de necesidad humana e, incluso, el de derecho social y el de igualdad de oportunidades sociales, irrenunciables para la dignificación de la persona y para una educación integral de la misma (Caride, 1998; Lull, 2001).

Y todo ello teniendo los ámbitos locales como referencia, pues son el lugar estratégico para la población en general pero, de manera particular, para los adolescentes. En ellos, los adolescentes, encuentran la estructura territorial en la que sentirse identificados y a partir de la cual poder promover y activar la participación, la autorrelización, la implicación y el compromiso respecto de su tiempo libre individual y colectivo, en el desarrollo de su ser persona y de su ser ciudadano (Sarrate, 2007). De ahí el necesario acercamiento a investigaciones como la que presentamos, centrada en ámbitos territoriales concretos en base a los cuales poder potenciar los valores que a través de la educación debemos inculcar en, para y mediante el tiempo libre de los adolescentes.

Se trata, en definitiva, de defender un concepto de educación en el ocio y en el tiempo libre dentro de un concepto de educación integral, cuyo ob- 
jetivo es poner en disposición a la persona para que conozca y tome decisiones respecto a su tiempo libre y a los valores que lo sostienen. Se trata de defender un concepto de educación en el tiempo libre donde la persona, individual y colectiva, personal y comunitaria, ejerce sus libertades y responsabilidades frente a un tiempo liberado de obligaciones, considerando los valores de fondo que mueven toda experiencia humana en cuanto que motor que capacita a las personas para mejorar sus vidas (Cuenca, 2004).

\section{METODOLOGÍA Y DISEÑO DE INVESTIGACIÓN}

La metodología de investigación seleccionada ha sido no experimental, ex post facto, descriptivo-correlacional, ya que no modificamos el objeto de estudio, no intervenimos directamente sobre las variables, simplemente registramos sus medidas (Arnal et al, 1992; Kerlinger y Lee, 2002). A través de estudios de encuesta (Kerlinger y Lee, 2002) centrando nuestra atención en el ocio y tiempo libre de los adolescentes, concretamente, en el ocio y tiempo libre de los adolescentes de Salamanca y provincia, el instrumento de recogida de datos de naturaleza cuantitativa, se basa en la metodología de encuestación estructurada.

El objetivo de este estudio ha tenido una doble orientación, por un lado, mostrar cuál es el índice de aprovechamiento del tiempo libre y los valores que sustentan dicha utilización, para comprobar si podemos hablar de un tiempo marcado por el ocio o, por el contrario, estamos hablando de un tiempo definido sin ninguna finalidad en sí misma, estéril, pasivo y tedioso. Y, por otro, analizar si las actividades llevadas a cabo bajo el rótulo de educativas tienen o no dicho carácter formativo.

\subsection{Instrumento: diseño y aplicación}

Fruto de la revisión de otros cuestionarios que ya existían en el mercado sobre diversos temas relacionados con el tiempo libre de los jóvenes elaboramos el cuestionario "El tiempo libre de los adolescentes en la provincia de Salamanca". Se estructura, a grandes rasgos, en tres apartados: el primero, relativo a preguntas sobre las actividades que en general se hacen en el tiempo libre, las razones que mueven a hacerlo y la frecuencia con que se realizan; el segundo, bloque central de la encuesta, está dividido en diversos apartados referentes a los temas concretos que queríamos analizar, - deportes, informática, televisión, lectura, drogas, familia y hogar, noche, amigos y música-; y el tercer bloque es el relacionado con las variables de clasificación, —localidad, edad, género, nivel de estudios, etc.—. Estos apartados vienen completados por algunas preguntas de respuesta abierta en tor- 
no a qué le gustaría tener o hacer en su tiempo libre que no tiene o no hace, o qué necesita para usar mejor su tiempo libre; y por otras preguntas de respuesta cerrada sobre la disponibilidad de tiempo libre y el grado de satisfacción respecto del mismo.

Una vez elaborado el cuestionario, procedimos a su validación aplicándolo a cuatro expertos en Pedagogía del tiempo libre, cuatro expertos en Metodología de la investigación educativa, cuatro alumnos, uno de cada curso, y cuatro padres de alumnos para que incluyesen las observaciones que estimasen oportunas antes de su elaboración definitiva. Una vez obtenidas las respuestas se procedió a su registro con la ayuda del paquete estadístico SPSS v.14.04; y, por último, llevamos a cabo los análisis estadísticos descriptivos e inferenciales oportunos.

\subsection{Participantes: obtención de muestra y perfil}

La población objeto de estudio la componen estudiantes de $1^{\circ}, 2^{\circ}, 3^{\circ}$ y $4^{\circ}$ de la ESO, escolarizados en centros públicos y privados, y ubicados en zonas rurales y urbanas. Para establecer el tamaño de la muestra solicitamos los datos de la población a la Dirección Provincial de Salamanca, Servicio de Planificación, obteniendo una población total de alumnos por sujetos (16.943), centros (182) y unidades (728) en el curso 2005-2006. Se llevó a cabo un muestreo aleatorio simple de unidades, cuyos puntos de muestreo se establecieron a partir de un listado de unidades, por centros escolares y estratos; se extrajeron las unidades que deberían formar parte de la muestra, mediante muestreo aleatorio simple. El procedimiento de muestreo se hizo por conglomerados y los centros se estratificaron según su localización geográfica (rural, urbana) y según su pertenencia al sector público o privado. En la tabla siguiente, se puede observar la configuración de la población y la muestra invitada extraída, con el fin de mantener la proporcionalidad de tipos de centro (ubicación y titularidad) y el resultado del muestreo de unidades escolares por centro. 


\begin{tabular}{|c|c|c|c|c|c|c|}
\hline & \multicolumn{3}{|c|}{ Población } & \multicolumn{3}{c|}{ Muestra } \\
\cline { 2 - 7 } & rural & urbano & totales & rural & urbano & totales \\
\hline Público & $\begin{array}{c}99 \\
(94,28 \%)\end{array}$ & $\begin{array}{c}53 \\
(69,73 \%)\end{array}$ & $\begin{array}{c}152 \\
(83,98 \%)\end{array}$ & $\begin{array}{c}5 \\
(83.33 \%)\end{array}$ & $\begin{array}{c}3 \\
(75,00 \%)\end{array}$ & $\begin{array}{c}8 \\
(80.00 \%)\end{array}$ \\
\hline Privado & 6 & 23 & 29 & 1 & 1 \\
$(5,72 \%)$ & $(30,27 \%)$ & $(16,02 \%)$ & $(16.66 \%)$ & $\begin{array}{c}2 \\
(25,00 \%)\end{array}$ & $(20.00 \%)$ \\
\hline Totales & 105 & $\begin{array}{c}76 \\
(100 \%)\end{array}$ & $\begin{array}{c}181 \\
(100 \%)\end{array}$ & $\begin{array}{c}6 \\
(100 \%)\end{array}$ & $\begin{array}{c}4 \\
(100 \%)\end{array}$ & $\begin{array}{c}10 \\
(100 \%)\end{array}$ \\
\hline
\end{tabular}

Tabla 1. Obtención de la muestra

Cruzando estos datos se obtienen cuatro estratos con sus respectivos centros: Público/rural ( 5 centros); Público/urbano (3 centros); Privado/rural (1 centro); Privado/urbano ( 1 centro), aportando un total de 10 centros, resultando, una vez pasados los cuestionarios, un muestra de 725 alumnos. Todo ello con un error muestral de 3\% (hipótesis de $p=50 \%$ y nivel de confianza del 95\%) (Santos et al, 2004).

De los 725 participantes, $61,5 \%(n=446)$ pertenecen a zonas rurales, $\mathrm{y}$ el $38,5 \%$ restante $(n=279)$ a zonas urbanas. Como era de esperar, la edad media se sitúa en los 14 años. El mayor número de sujetos se sitúa entre los 13 y los 15 años, y a partir de los 16 va decreciendo la cifra, siendo tan solo 5 alumnos los que tienen 18 años y un caso aislado con 20. Un grupo que, como tal, señala diferencias claras que dificultan su estudio en cuanto grupo; unos no han hecho más que dejar la niñez y otros se sitúan próximos a la juventud. En relación al género existe un porcentaje similar entre hombres y mujeres, siendo algo mayor el de mujeres $(53,4 \%)$ frente al de hombres $(46,6 \%)$. Respecto al nivel de estudios, entre los alumnos de $2^{\circ}$ y $4^{\circ}$ de la ESO existe un cierto desnivel en el número de alumnos, inclinándose la balanza en favor de los alumnos de $2^{\circ}$ de la ESO, con mayor número, $210(29,0 \%)$ frente a los 147 de $4^{\circ}(20,3 \%)$. Y, por último, el porcentaje de alumnos por centro es similar, giran todos entre el 9 y $11 \%$.

\section{ANÁLISIS DE DATOS Y RESULTADOS}

Nuestra pretensión más allá de conocer qué papel atribuyen los adolescentes, en momentos de ocio, a la familia, los amigos, al deporte, la lectura, la música o el ocio nocturno, trata de constatar si la edad, considerada a través del nivel educativo en el que se encuentran los participantes $\left(1^{\circ}, 2^{\circ}\right.$, 
$3^{\circ}$ y $4^{\circ}$ ESO) es un factor que condiciona la práctica de dichas actividades, así como la visión de las mismas. En cuanto a las distintas actividades enumeradas tratamos de obtener información sobre si se practican o no, sobre cuál/es practican, la frecuencia y el motivo. Nos hemos detenido en los resultados más significativos sin entrar en todos y cada uno de los apartados de los que constaba la encuesta, centrándonos, en primer lugar, en aspectos relacionales, -familia y grupo de iguales_-, para, en segundo lugar, constatar las diferencias en las principales actividades que dan cuerpo a su tiempo libre, emparejándolas sin ningún criterio relacional sino, más bien, por mera cuestión de presentación.

\subsection{Familia y grupo de iguales}

En relación al tiempo libre que los adolescentes encuestados pasan en familia, los estudiantes de $1^{\circ}$ manifiestan dedicar más tiempo que el resto de los grupos, existiendo diferencias significativas $(F=4,159 ; p=0,006)$ entre el grupo de $1^{\circ}$ y el de $3^{\circ}$, que dedica menos tiempo libre a la familia (como puede verse en la tabla 2 ).

\begin{tabular}{|c|c|c|c|c|c|c|c|c|c|c|c|c|c|}
\hline & \multicolumn{2}{|c|}{$\mathbf{1}^{\mathbf{o}}$ ESO } & \multicolumn{2}{c|}{$\mathbf{2}^{\mathbf{o}}$ ESO } & \multicolumn{2}{c|}{$\mathbf{3}^{\mathbf{o}}$ ESO } & \multicolumn{2}{|c|}{$\mathbf{4}^{\mathbf{o}}$ ESO } & \multicolumn{2}{|c|}{ Total } & \multicolumn{2}{|c|}{ ANOVA } & Dif. \\
\cline { 2 - 12 } & $\bar{X}$ & $S x$ & $\bar{X}$ & $S x$ & $\bar{X}$ & $S x$ & $\bar{X}$ & $S x$ & $\bar{X}$ & $S x$ & $F$ & $p$ & \\
\hline $\begin{array}{c}\text { ¿Cuánto tiempo } \\
\text { libre pasas con } \\
\text { tu familia? }\end{array}$ & 3,67 & 0,970 & 3,61 & 1,006 & 3,34 & 0,979 & 3,48 & 0,931 & 3,52 & 0,982 & 4,159 & 0,006 & $1^{\circ}-3^{\circ}$ \\
\hline
\end{tabular}

Tabla 2. Análisis de varianza, por niveles educativos, tiempo libre con la familia

En cambio, no difieren en cuanto al grado de satisfacción durante el tiempo libre que pasan familiarmente, mostrándose todos bastante satisfechos al respecto $(F=0.652 ; p=0.582)$. Aunque la relación con los padres cambia cuantitativamente, en cuanto que los adolescentes ya no están tantas horas en casa, también se produce un cambio cualitativo, en la medida en que aunque hablan más de sus problemas con los amigos, siguen necesitando a sus padres a la hora de formar sus valores.

Respecto al grupo de iguales, la proporción de adolescentes que afirman o niegan salir diariamente con los amigos, es similar, no existiendo diferencias significativas entre niveles educativos (chi cuadrado $=2,848$; $p=0,416)$. 


\begin{tabular}{|c|c|c|c|c|c|c|c|c|c|c|c|c|}
\hline \multirow{2}{*}{$\begin{array}{c}\text { Sales a diario con los } \\
\text { amigos }\end{array}$} & \multicolumn{2}{|c|}{$1^{\circ} \mathrm{ESO}$} & \multicolumn{2}{|c|}{$2^{\circ} \mathrm{ESO}$} & \multicolumn{2}{|c|}{$3^{\circ}$ ESO } & \multicolumn{2}{|c|}{$4^{\circ} \mathrm{ESO}$} & \multicolumn{2}{|c|}{ Total } & \multicolumn{2}{|c|}{ Diferencias } \\
\hline & $\mathrm{f}$ & $\%$ & $\mathrm{f}$ & $\%$ & $\mathrm{f}$ & $\%$ & $\mathrm{f}$ & $\%$ & $\mathrm{f}$ & $\%$ & $X^{2}$ & $p$ \\
\hline Sí & 92 & 53,8 & 110 & 53,4 & 91 & 47,9 & 68 & 46,6 & 361 & 50,6 & 2,848 & 0,416 \\
\hline No & 74 & 46,2 & 96 & 46,6 & 99 & 52,1 & 78 & 53,4 & 352 & 49,4 & & \\
\hline Total & 171 & 100 & 206 & 100 & 190 & 100 & 146 & 100 & 713 & 100 & & \\
\hline
\end{tabular}

Tabla 3. Chi cuadrado, salir con los amigos a diario

No obstante, en $1^{\circ}$ y $2^{\circ}$ es ligeramente más elevado el grupo de estudiantes que afirma salir a diario con sus amigos, 53,8\% y 53,4\% respectivamente, no siendo así en $3^{\circ}(47,9 \%)$ y $4^{\circ}(46,6 \%)$, en donde la tendencia se invierte. Tampoco se dan diferencias en función del nivel educativo, en lo que concierne a los fines de semana (chi cuadrado $=1,687 ; p=0,640$ ), observando una tendencia mayoritaria a las salidas habituales durante este periodo, superando en todos los casos el 94\%.

\begin{tabular}{|c|c|c|c|c|c|c|c|c|c|c|c|c|}
\hline \multirow{2}{*}{$\begin{array}{c}\text { Sales los fines } \\
\text { de semana con los } \\
\text { amigos }\end{array}$} & \multicolumn{2}{|c|}{$1^{\circ} \mathrm{ESO}$} & \multicolumn{2}{|c|}{$2^{\circ}$ ESO } & \multicolumn{2}{|c|}{$3^{\circ} \mathrm{ESO}$} & \multicolumn{2}{|c|}{$4^{\circ} \mathrm{ESO}$} & \multicolumn{2}{|c|}{ Total } & \multicolumn{2}{|c|}{ Diferencias } \\
\hline & $\mathrm{f}$ & $\%$ & $\mathrm{f}$ & $\%$ & $\mathrm{f}$ & $\%$ & $\mathrm{f}$ & $\%$ & $\mathrm{f}$ & $\%$ & $X^{2}$ & $p$ \\
\hline Sí & 158 & 92,4 & 194 & 93,7 & 179 & 95,2 & 139 & 98,2 & 670 & 94,1 & 1,68 & 0,640 \\
\hline No & 13 & 7,6 & 13 & 6,3 & 9 & 4,8 & 7 & 4,8 & 42 & 5,9 & & \\
\hline Total & 171 & 100 & 207 & 100 & 188 & 100 & 146 & 100 & 712 & 100 & & \\
\hline
\end{tabular}

Tabla 4. Chi cuadrado, salir con los amigos los fines de semana

Entre las actividades que más realizan los adolescentes de la muestra cuando están con sus amigos, destacan en este orden, las de hablar, ir a casa de algún amigo, sentarse en una plaza o calle, pasear, escuchar música, jugar, ir al cine, hacer deporte, ir de bares o discotecas y beber. No obstante, hemos de señalar que jugar es una de las actividades realizadas mayoritariamente por los alumnos de $1^{\circ}$ y $2^{\circ}$, al igual que hacer deporte, mientras que beber, fumar, escuchar música o ir de bares son actividades más efectuadas por los alumnos de $3^{\circ}$ y $4^{\circ}$. 


\begin{tabular}{|c|c|c|c|c|c|c|c|c|c|c|c|c|}
\hline \multirow{2}{*}{$\begin{array}{c}\text { ¿Cuáles de estas } \\
\text { actividades haces } \\
\text { cuando estás con los } \\
\text { amigos? }\end{array}$} & \multicolumn{2}{|c|}{$1^{\circ}$ ESO } & \multicolumn{2}{|c|}{$2^{\circ}$ ESO } & \multicolumn{2}{|c|}{$3^{\circ}$ ESO } & \multicolumn{2}{|c|}{$4^{\circ}$ ESO } & \multicolumn{2}{|c|}{ Total } & \multicolumn{2}{|c|}{ Diferencias } \\
\hline & $\mathrm{f}$ & $\%$ & $\mathrm{f}$ & $\%$ & $\mathrm{f}$ & $\%$ & $\mathrm{f}$ & $\%$ & $\mathrm{f}$ & $\%$ & $X^{2}$ & $p$ \\
\hline Hablar & 148 & 86,0 & 179 & 86,1 & 168 & 88,0 & 136 & 92,5 & 631 & 87,9 & 4,161 & 0,245 \\
\hline Pasear & 101 & 58,7 & 122 & 58,7 & 117 & 61,3 & 105 & 71,4 & 445 & 62,0 & 7,363 & 0,061 \\
\hline Jugar & 131 & 76,2 & 126 & 60,6 & 84 & 44,0 & 73 & 49,7 & 414 & 57,7 & 43,342 & 0,000 \\
\hline Beber & 29 & 16,9 & 58 & 27,9 & 88 & 46,1 & 69 & 46,9 & 244 & 34,0 & 49,368 & 0,000 \\
\hline Fumar & 16 & 9,3 & 34 & 16,3 & 45 & 23,6 & 27 & 18,4 & 122 & 17,0 & 13,312 & 0,009 \\
\hline Escuchar música & 93 & 54,1 & 125 & 60,1 & 135 & 70,7 & 91 & 61,9 & 444 & 61,8 & 10,995 & 0,012 \\
\hline Ir al cine & 103 & 59,9 & 122 & 58,7 & 93 & 48,7 & 84 & 57,1 & 402 & 56,0 & 5,886 & 0,118 \\
\hline Ir a bares y discotecas & 27 & 15,7 & 71 & 34,1 & 118 & 61,8 & 104 & 70,7 & 320 & 44,6 & 130,882 & 0,000 \\
\hline $\begin{array}{l}\text { Sentarnos en la plaza } \\
\text { o calle }\end{array}$ & 105 & 61,0 & 129 & 62,0 & 115 & 60,2 & 96 & 65,3 & 445 & 62,0 & 1,008 & 0,799 \\
\hline Ir de botellón & 25 & 14,5 & 57 & 27,4 & 71 & 37,2 & 61 & 41,5 & 214 & 29,8 & 34,303 & 0,000 \\
\hline Deporte & 107 & 62,2 & 104 & 50,0 & 83 & 43,5 & 68 & 46,3 & 362 & 50,4 & 14,302 & 0,003 \\
\hline $\begin{array}{l}\text { Buscar bronca con } \\
\text { otros }\end{array}$ & 21 & 12,2 & 30 & 14,4 & 27 & 14,2 & 11 & 7,5 & 89 & 12,4 & 4,630 & 0,201 \\
\hline
\end{tabular}

Tabla 5. Chi cuadrado. Actividades realizadas con los amigos

\subsection{Ocio nocturno y Deporte}

Las salidas nocturnas durante el fin de semana, constituye una actividad bastante habitual entre el colectivo de adolescentes, a la que dedican una parte importante de su tiempo libre.

Tal como cabía esperar los alumnos de $3^{\circ}$ y $4^{\circ}$ son los que en mayor medida salen por las noches los fines de semana observándose diferencias significativas respecto a sus compañeros de $1^{\circ}$ y $2^{\circ}($ chi cuadrado $=58,490$; $p=0,000)$.

\begin{tabular}{|c|c|c|c|c|c|c|c|c|c|c|c|c|}
\hline \multirow{2}{*}{$\begin{array}{l}\text { ¿Sales por las noches } \\
\text { los fines de semana } \\
\text { (a partir de las 10)? }\end{array}$} & \multicolumn{2}{|c|}{$1^{\circ}$ ESO } & \multicolumn{2}{|c|}{$2^{\circ}$ ESO } & \multicolumn{2}{|c|}{$3^{\circ}$ ESO } & \multicolumn{2}{|c|}{$4^{\circ} \mathrm{ESO}$} & \multicolumn{2}{|c|}{ Total } & \multicolumn{2}{|c|}{ Diferencias } \\
\hline & $\mathrm{f}$ & $\%$ & $\mathrm{f}$ & $\%$ & $\mathrm{f}$ & $\%$ & $\mathrm{f}$ & $\%$ & $\mathrm{f}$ & $\%$ & $X^{2}$ & $p$ \\
\hline Sí & 68 & 40,0 & 105 & 51,7 & 136 & 72,3 & 109 & 74,7 & 418 & 59,1 & 58,490 & 0,000 \\
\hline No & 102 & 60,0 & 98 & 48,3 & 52 & 27,7 & 37 & 25,3 & 289 & 40,9 & & \\
\hline Total & 170 & 100 & 203 & 100 & 188 & 100 & 146 & 100 & 707 & 100 & & \\
\hline
\end{tabular}

Tabla 6. Chi cuadrado. Salida por las noches los fines de semana. 
En cuanto a la hora de llegada, en buena parte de los casos, se produce entre las 11 y la 1 de la madrugada, aunque es importante la proporción de jóvenes de $2^{\circ}, 3^{\circ}$ y $4^{\circ}$ que manifiestan, comparándolos con los de $1^{\circ}$, volver a casa entre las 3 y las 5 , encontrándose, por tanto, diferencias significativas ( chi cuadrado $=46,094 ; p=0,000$ ).

No hemos podido constatar diferencias interniveles en cuanto a si prefieren llegar más tarde de lo que lo hacen (chi cuadrado $=0,456 p=0,747$ ), aunque si nos fijamos en los porcentajes totales, la tendencia generalizada es que sí les gustaría (63,4\% afirma que sí frente al 36,6\% que no).

El hecho de salir de noche adquiere diferencias significativas interniveles (chi cuadrado $=52,080 ; p=0,000$ ); así supone para los adolescentes de modo especial una sensación de libertad y de hacer algo diferente, aunque en el caso del grupo de $1^{\circ}$, la sensación de libertad es la razón más significativa $(43,7 \%)$; mientras que para los estudiantes de $4^{\circ}$, el salir de noche les produce más la sensación de hacer algo diferente (50,8\%).

El 78,4\% de los encuestados afirman practicar algún deporte, formando parte de la dinámica habitual de relación y convivencia entre los participantes del estudio. Los motivos más destacados para practicar algún deporte son los de hacer ejercicio y mantenerse en forma (55,6\%), divertirse $(54,2 \%)$, el gusto por un deporte en concreto (52,2\%), superando en todos los casos el $40 \%$, no existiendo diferencias interniveles en dichas razones. Sí se dan, sin embargo, en lo que concierne a la práctica de deportes por el hecho de estar con los amigos (chi cuadrado =10,431; $p=0,015$ ), de tal forma que los alumnos de $1^{\circ}(47,9 \%)$ y $2^{\circ}(46,7 \%)$ de ESO son los que en mayor medida manifiestan aducir estas razones.

\subsection{Ordenador y televisión}

La mayor parte de los adolescentes encuestados emplean el ordenador para escuchar/bajar música $(70,5 \%)$, jugar $(62,1 \%)$, chatear $(61,4 \%)$, hacer trabajos $(59,5 \%)$, pasar el rato $(48,1 \%)$, ver/bajar películas $(47,2 \%)$, o ver el correo electrónico $(46,9 \%)^{5}$.

No obstante, en nuestro estudio encontramos algunas diferencias, de modo que los alumnos de $1^{\circ}$ y de $4^{\circ}$ (chi cuadrado $=10,278 ; p=0,016$ ), por ejemplo, utilizan más el ordenador para hacer trabajos que los demás grupos; los de $1^{\circ}$ y $2^{\circ}$ manifiestan emplearlo en mayor medida que los restantes alumnos para jugar (chi cuadrado $=8,390 ; p=0,039$ ). Los estudiantes de $2^{\circ}$, $3^{\circ}$ y $4^{\circ}$ se decantan sobre todo por el empleo del ordenador para escuchar música o bajársela de Internet (chi cuadrado $=11,131 ; p=0,011$ ). 


\begin{tabular}{|l|c|c|c|c|c|c|c|c|c|c|c|c|c|}
\hline \multirow{2}{*}{$\begin{array}{c}\text { ¿Para qué utilizas } \\
\text { el ordenador? }\end{array}$} & \multicolumn{2}{|c|}{$\mathbf{1}^{\mathbf{o}}$ ESO } & \multicolumn{2}{c|}{$\mathbf{2}^{\mathbf{o}}$ ESO } & \multicolumn{2}{c|}{$3^{\mathbf{0}}$ ESO } & \multicolumn{2}{c|}{$\mathbf{4}^{\mathbf{0}}$ ESO } & \multicolumn{2}{|c|}{ Total } & \multicolumn{2}{c|}{ Diferencias } \\
\cline { 2 - 15 } & $\mathrm{f}$ & $\%$ & $\mathrm{f}$ & $\%$ & $\mathrm{f}$ & $\%$ & $\mathrm{f}$ & $\%$ & $\mathrm{f}$ & $\%$ & $X^{2}$ & $p$ \\
\hline Chatear & 110 & 63,2 & 117 & 55,7 & 128 & 66,7 & 89 & 60,5 & 444 & 61,4 & 5,400 & 0,145 \\
\hline Navegar por la Red & 53 & 30,5 & 54 & 25,7 & 66 & 34,4 & 52 & 35,4 & 225 & 31,1 & 5,088 & 0,165 \\
\hline $\begin{array}{l}\text { Ver el correo } \\
\text { electrónico }\end{array}$ & 81 & 46,6 & 84 & 40,0 & 99 & 51,6 & 75 & 51,0 & 339 & 46,9 & 6,701 & 0,082 \\
\hline Pasar el rato & 79 & 45,4 & 102 & 48,6 & 88 & 45,8 & 79 & 53,7 & 348 & 48,1 & 2,795 & 0,424 \\
\hline Hacer trabajos & 105 & 60,3 & 113 & 53,8 & 109 & 56,8 & 103 & 70,1 & 430 & 59,5 & 10,278 & 0,016 \\
\hline Jugar & 121 & 69,5 & 135 & 64,3 & 110 & 57,3 & 83 & 56,5 & 449 & 62,1 & 8,390 & 0,039 \\
\hline $\begin{array}{l}\text { Conocer a otras } \\
\text { personas }\end{array}$ & 34 & 19,5 & 34 & 16,2 & 28 & 14,6 & 25 & 17,0 & 121 & 16,7 & 1,673 & 0,643 \\
\hline $\begin{array}{l}\text { Escuchar/bajar } \\
\text { música }\end{array}$ & 108 & 62,1 & 148 & 70,5 & 138 & 71,9 & 116 & 78,9 & 510 & 70,5 & 11,131 & 0,011 \\
\hline Ver/bajar películas & 71 & 40,8 & 100 & 47,6 & 97 & 50,5 & 73 & 49,7 & 341 & 47,2 & 4,077 & 0,253 \\
\hline
\end{tabular}

Tabla 7. Chi cuadrado. ¿Para qué lo utilizas? (el ordenador)

Referente a si les gusta o no ver la televisión, los resultados muestran que a la mayoría $(69,2 \%)$ sí. Entre las razones no se dan diferencias estadísticamente significativas entre los diferentes grupos. Tan sólo resaltar que un $68,0 \%$ de la muestra considera que lo hace por gusto, y un $23,9 \%$ para no aburrirse. En cuanto a la frecuencia con la que se ve, predominan quienes afirman verla entre 1 y 2 horas (40\%), aunque también cabe especificar aquellos que la ven más de dos horas $(39,7 \%)$, independientemente del nivel en el que se encuentren (chi cuadrado $=8,976 ; p=0,439$ ).

Los resultados ponen de manifiesto que los programas más vistos son, en este orden, las películas, deportes, dibujos, concursos, teleseries, musicales e informativos. Los programas rosas y los realities parecen ser, tal como muestran los resultados, los programas más vistos por los alumnos de $3^{\circ}\left(42,7 \%\right.$ y $46,9 \%$, respectivamente) y $4^{\circ}(50,3 \%$ y $54,4 \%$ respectivamente). Los dibujos, deportes y documentales son preferidos fundamentalmente por el alumnado de $1^{\circ}(58,6 \%, 59,8 \%$ y $31,6 \%$ respectivamente). Los programas deportivos también son del agrado del grupo de $2^{\circ}(59,8 \%)$. Por tanto, la edad es también determinante a la hora de seleccionar los programas televisivos. 


\subsection{Lectura y Música}

En el estudio efectuado, y teniendo en cuenta el nivel educativo, no hemos encontrado diferencias entre los grupos cuando se les pregunta si les gusta leer $(F=1,885 ; p=0,131)$, observándose en todos los casos una tendencia a considerar que la lectura no es una de sus actividades preferidas ${ }^{6}$.

Si atendemos, sin embargo, a los periodos en los que leen, durante la semana o el fin de semana se observan diferencias, de tal forma que los alumnos de $1^{\circ}$ manifiestan leer con mayor frecuencia durante la semana y en el fin de semana que los de $3^{\circ}(F=3,994 ; p=0,008$; durante la semana y $F=2,863 ; p=0,036$, fines de semana), no existiendo diferencias entre el resto de grupos.

Aunque en los diferentes niveles educativos considerados la tendencia de los adolescentes es la de manifestar que cuando leen lo hacen por gusto $(42,6 \%)$, hemos apreciado algunas diferencias en los análisis estadísticos efectuados. Así por ejemplo, destacan los porcentajes de alumnos de $3^{\circ}$ y $4^{\circ}$ que afirman leer por gusto (44,7\% y 47,8\%, respectivamente), si los comparamos con los de sus compañeros más jóvenes $\left(41.9 \%, 37.4 \%, 1^{\circ}\right.$ y $2^{\circ}$ respectivamente).

Quizá el hecho de que sean más maduros y con mayor capacidad para tomar decisiones explique este dato. Es destacable el hecho de que en el último nivel $\left(4^{\circ}\right)$ encontremos mayores porcentajes de respuesta, considerando los restantes grupos, en la opción por trabajo (20,3\%); es un dato que, por otra parte, no ha de extrañarnos si tenemos en cuenta que las exigencias académicas son más elevadas a medida que vamos avanzando por los distintos niveles educativos.

En alusión a la música, la mayoría dedican más de dos horas a escuchar música diariamente, se aprecian diferencias interniveles (chi cuadrado $=32,223 ; p=0,000)$, de modo que los estudiantes de $1^{\circ}$ y $2^{\circ}$ dedican menos de una hora, mientras que los de $3^{\circ}$ y $4^{\circ}$ invierten más tiempo. En todos los casos el tiempo aumenta durante el fin de semana, que lógicamente, la disposición de tiempo libre es mayor. Así, el 50,6\% del alumnado escuchan música entre 1 y 3 horas los fines de semana, aunque en este caso, la tendencia se invierte aunque siguen manteniéndose diferencias significativas (chi cuadrado $=28,706 ; p=0,001$ ). Los alumnos de $1^{\circ}$ y $2^{\circ}$ tienden a escuchar más música que los de $3^{\circ}$ y $4^{\circ}$.

En cuanto al tipo de música que escuchan destaca la música pop, jazz, instrumental, tecno y disco, no existiendo diferencias interniveles en cuanto a sus preferencias musicales, en ninguno de los casos, n.s. 0,057. 


\section{CONCLUSIONES Y DISCUSIÓN}

De acuerdo con la revisión efectuada, a partir de la investigación que hemos llevado a cabo, podemos afirmar que existen, en términos generales, diferencias significativas en función del nivel educativo entre los alumnos de la ESO en Salamanca y provincia respecto a su forma de entender y de llenar su tiempo libre. Las diferencias existentes, principalmente, entre los cursos de $1^{\circ}$ y $2^{\circ}$ respecto de $3^{\circ}$ y $4^{\circ}$ dejan patente que las diferencias que puedan encontrarse en las aulas entre los chicos de 11 a los de 13 años mantienen su prolongación en el tiempo libre, a la luz de los resultados, por ejemplo hay diferencias en función del nivel entre los alumnos de $1^{\circ}$ y $3^{\circ}$ en el tiempo que pasan con la familia; o entre los alumnos de $3^{\circ}$ y $4^{\circ}$ con respecto a los de $1^{\circ}$ y $2^{\circ}$ cuando preguntamos acerca de las salidas durante los fines de semana... "Lograr la cuadratura del círculo o, lo que es lo mismo, responder a la heterogeneidad del alumnado..." (Romero et al, 2009, 113), encuentra procesos análogos en los tiempo libres de los chicos.

Desde un contexto general y sin entrar en diferencias interniveles, los valores que mueven el tiempo libre de los adolescentes son más bien escasos desde un punto de vista educativo. Es la única vía a través de la que poder tener un análisis real de la situación educativa que se esconde tras la práctica de unas u otras actividades, más aún cuando hemos comprobado que algunas de las actividades tildadas de educativas esconden un bajo índice formativo en los adolescentes. La falta de libertad de los adolescentes a la hora de elegir, el deseo de los padres de que hagan los hijos lo que ellos no pudieron hacer, la sobresaturación de actividades — deportes, música, idiomas, etc.-, pueden hacer perder el objetivo con el que fueron elegidas esas actividades para ocupar el tiempo libre, dejando un claro vacío educativo.

Entrando en detalles y en relación a las diferencias interniveles, las conclusiones a las que hemos llegado encuadran a los adolescentes salmantinos como sujetos que practican deporte habitualmente $(78,3 \%)$, si bien en los dos primeros cursos se ve el valor del grupo por encima de la actividad que realizan. Los adolescentes son conscientes de que en los dos primeros cursos de la etapa se consolida el grupo como núcleo de referencia relacional y el deporte pone las condiciones necesarias para afianzarse dentro de él. El Deporte sigue siendo una de las actividades centrales del adolescente en su tiempo libre.

Y en términos similares nos encontramos con el tiempo dedicado a la noche. Los adolescentes de los dos primeros cursos no sólo salen menos sino, sobre todo, el valor que le dan a la noche es notoriamente distinto pues si los mayores buscan hacer algo distinto los más jóvenes, aquellos que están despertando a nuevas sensaciones, se apoyan en el valor de la libertad y en la 
sensación que les produce. Dato que se debería tener en cuenta a la hora de gestionar cualquier programa alternativo de ocio que surja en el contexto de la noche.

En lo que atañe a la familia de nuevo observamos cómo los adolescentes más jóvenes siguen teniendo pautas similares en el tiempo libre y en las actividades de ocio a las manifestadas durante la infancia que los mayores, observando un grado de satisfacción al estar con la familia. Si bien buscan el grupo como referencia directa, el nicho primario, aunque desde un segundo plano, sigue siendo la familia, principalmente entre los más jóvenes.

En cambio, como acabamos de señalar, el grupo de iguales es el ámbito de encuentro, principalmente en los más pequeños que buscan dar el salto de la familia al grupo, debido, probablemente, bien a que las responsabilidades de tipo académico conllevan más tiempo de dedicación a los mayores, bien a que el grupo está hecho y no necesitan verse tanto para consolidarse en el mismo.

Respecto a la lectura hemos comprobado que sigue siendo un tema pendiente. Se lee poco en el tiempo libre, y en los fines de semana menos aún, pues están saturados con otro tipo de actividades que tienen más que ver con las relaciones, las personas y la calle. No obstante, da la impresión de que los adolescentes quieren mostrar un cierta actitud positiva hacia la lectura; con todo, sigue existiendo un porcentaje de no lectores bastante significativo (nada el 17,3\%, muy poco, $18,8 \%$ y algo el $32,0 \%$ ) y no resulta copioso el colectivo de lectores habituales $(13,1 \%)$. Y analizando las diferentas interniveles hemos comprobado que a medida que avanzan en edad leen menos, principalmente en el tercer curso debido, quizá, a que el hábito lector no ha entrado aún en competencia, como ya hemos señalado, con actividades de ocio más atractivas para adolescentes de mayor edad. No obstante ese porcentaje menor en los adolescentes mayores se ve enriquecido cuando vemos que el valor que sostiene dicha actividad es el gusto y no la obligación, lo que se traduce en el hecho de que es en los dos últimos cursos cuando podemos empezar a hablar de lectores habituales y maduros.

La música, por su parte, es otra de las actividades preferidas por los adolescentes para llevar a cabo en su tiempo libre. Los alumnos de la ESO pueden ser considerados oyentes habituales de música, tanto a diario como los fines de semana. Las diferencias encontradas interniveles de nuevo nos muestran diferencias entre los de $1^{\circ}$ y $2^{\circ}$ y los de $3^{\circ}$ y $4^{\circ}$. Resulta claro que el hecho de escuchar música va asociado al tiempo que dejan libre otro tipo de actividades, pues los fines de semana es cuando los más jóvenes escuchan más música, pues al no tener asentado el grupo y las actividades que llevan a cabo en su seno, disponen de más tiempo para llevar a cabo esta actividad. 
El uso del ordenador va asociado a la conexión a Internet y a las posibilidades que la Red despliega para los jóvenes. En este sentido bajar música y ver películas son dos de las actividades más practicadas, junto con jugar y chatear. Los más pequeños son quienes nuevamente lo utilizan preferentemente para jugar mientras que en los mayores predomina su uso para bajar, escuchar música y chatear. El tiempo invertido en el ordenador cada vez es mayor, siendo conscientes de que buena parte de su uso viene descrito en términos de relación interpersonal. Es en esta edad cuando las relaciones entre los chicos conviven de forma análoga en los espacios tradicionales y en los espacios virtuales.

La televisión sigue siendo uno de los ejes principales en el tiempo libre de los adolescentes. La mayor parte ven la televisión porque les gusta $(68.0 \%)$. No existen grandes valores bajo el tiempo dedicado a la televisión más allá de no aburrirse y estar viendo algo que resulte entretenido. Respecto a posibles diferencias significativas de nuevo las encontramos entre los más pequeños respecto de aquellos que están terminando la etapa obligatoria en lo que atañe a gustos por unos u otros tipos de programas, cuya explicación puede encontrarse en la libertad que los mayores pueden tener a la hora de escoger los programas, sin que medie tanto el control de los padres en dicha elección, como podría ocurrir en edades más tempranas.

Por último y respecto a la hipótesis planteada, concluimos que resulta difícil constatar, una vez aplicado el cuestionario, cuáles son las reflexiones de los adolescentes sobre los usos y la distribución de su tiempo libre, al igual que cuáles son sus pensamientos acerca de la existencia de diferentes opciones de vivir y aprovechar dicho tiempo. No obstante, y a la luz de los resultados obtenidos, es ineludible poner en evidencia la necesidad de nuevas propuestas y programas alternativos que ayuden a los adolescentes de hoy, a canalizar dicho tiempo.

Estas conclusiones particulares se transforman en inquietud que, entre otras, se concreta, en dos posibles ejes de discusión sobre los que fundamentar futuras propuestas de actuación. Un primer eje es el que alude a la necesidad de respetar y basar las actividades y alternativas en los grupos de referencia. Tal y como muestran datos en el epígrafe relativo a familia y grupo de iguales, con la entrada en el período de la adolescencia, la familia, incluso la escuela, aunque no dejan de ser los ejes prioritarios de soporte de la vida de los adolescentes, sí que dejan paso, al otorgarle el mismo valor, a los iguales. El grupo de iguales y los amigos son quienes ocupan esa zona de construcción de la persona y ese tiempo libre del que disponen con unos porcentajes amplios, — por ejemplo, más del 94\% de la muestra en los cuatro cursos afirma salir con los amigos los fines de semana-. Las relaciones interpersonales son y deben ser el detonante principal, no único, de cual- 
quier propuesta de actuación en el tiempo libre, más aún si tenemos en cuenta la relaciones líquidas (Bauman, 2007) bajo las que conviven los adolescentes hoy en día. Son agrupaciones informales, frágiles, en buena parte de los casos pero, intencionadas y vitales.

El deseo de libertad, de autonomía, de conocer cosas nuevas y con gente nueva, de saborear y descubrir todo tipo de experiencias distintas a las llevadas a cabo en el seno familiar, y todo ello pasando el mayor tiempo posible con sus iguales, deben en parte, atravesar cualquier propuesta, bien desde la música, el deporte, la Red, la lectura, etc. El grupo de amigos es su refugio y, como tal, es el ámbito donde anidan los valores, actitudes, aspectos cruciales en todo planteamiento de educación y tiempo libre. Unas veces para reforzarlos y otras para superarlos, los valores y los centros de interés que subyacen a su grupo y a las formas de invertir su tiempo deben ser punto de partida.

Y un segundo eje, más concreto y particular, se aferra a tres de los tópicos que deben tener y tienen cabida en la vida de un adolescente: la música, la imagen y la lectura. La primera y la segunda porque las practican mucho, - casi toda la muestra dedica más de dos horas a escuchar música a diario y buena parte de la muestra invierte el mismo tiempo en ver la televisión y estar frente a la pantalla del ordenador-, y la tercera porque está sobradamente demostrado su importante valor educativo (Bransford, Brown y Cocking, 2000; Etxeberría y otros, 2008). En este sentido y teniendo en cuenta el valor que otorgan a la música, pensamos que puede resultar de interés formativo para los adolescentes plantear la lectura y promover planes de fomento de la lectura en base a la música que les gusta escuchar y utilizando como soporte la imagen.

Y existen buenos ejemplos en el mercado que nos pueden servir de guía y modelo. Entre ellos podemos destacar los libros/cd que empiezan a tener un hueco en el mercado, o los libros/dvd donde explican el porqué de las canciones, de las letras, la vida del autor, de forma interactiva, resultando así más interesante para los adolescentes. En los tiempos actuales no sólo hay que leer sino también contextualizar la lectura y para ello, la música y la imagen tienen un valor crucial (Hormigos, 2010). La televisión e Internet pueden ser dos buenos apoyos de fomento de la lectura. 


\section{NOTAS}

1. Con un vistazo somero a la página Web de publicaciones del INJUVE podemos comprobar el alcance de las investigaciones que sobre juventud y tiempo libre se han llevado a cabo. http://www.injuve.mtas.es/injuve/contenidos.type.action?type $=1768363455 \&$ menuId=1768363455\&mimenu=Ediciones\%20Injuve [Consulta 2009, 8 de Octubre].

2. A ello han contribuido tanto el apoyo de las administraciones públicas como la proliferación de las asociaciones y colectivos encargados de ayudar a usufructuar los tiempos del mejor modo posible. Concretamente en Castilla y León se cuenta con la Dirección General de la Juventud, dependiente de la Consejería de Familia e Igualdad de Oportunidades, con numerosa legislación al respecto, en el que se encuentra igualmente el Instituto de la Juventud. Destacable resulta, en igual medida, el informe anual que publica la Junta de Castilla y León bajo el título "Situación económica y social de la juventud", en el que se muestra un análisis estadístico y social del panorama regional que presentan los jóvenes en nuestra región. Más concretamente, en el capítulo quinto, se analiza el reparto de tiempo, ocio, deporte, asociacionismo, entre otros aspectos.

3. "Análisis, diagnóstico y propuesta de mejora para el tiempo libre de los adolescentes en la provincia de Salamanca. Diferencias entre espacios rurales y urbanos". Investigación dirigida por el Dr. José Manuel Muñoz Rodríguez, y financiada por la Junta de Castilla y León, SA019B06-.

4. CIS (1997). Encuesta sobre tiempo libre y deportes. Disponible en http://www.cis.es; (1997). Tiempo libre e informática. Disponible en http://www.cis.es; (2003). Encuesta sobre tiempo libre y hábitos de lectura II. Disponible en http://www.cis.es; (2005). Sondeo sobre la juventud española. Disponible en http://www.cis.es; INJUVE (1997) Cambios de hábitos en el uso del ocio. Madrid, Instituto de la Juventud.; (2000). Ocio y tiempo libre: identidades y alternativas. Disponible en http://injuve.mtas.es; (2001). Sondeo periódico de opinión y situación de la gente joven. Consejería Técnica de Planificación y Evaluación. Servicio de Documentación y estudios. Disponible en http://injuve.mtas.es; (2002). Sondeo de opinión en torno al ocio y tiempo libre, noche y fin de semana, consumo de alcohol, tabaco y otras sustancias (Referencia EJ077/2002). Disponible en http://injuve.mtas.es; (2003). Ocio y tiempo libre: Noche y fin de semana. Salud y sexualidad. Disponible en http://injuve.mtas.es; (2004). Ocio y tiempo libre: noche y fin de semana, consumo de bebidas alcohólicas, inmigración. Disponible en http://injuve.mtas.es; ASECAL. (2005) Estudio sobre prácticas de ocio de los jóvenes de Peñaranda de Bracamonte. Documento fotocopiado. [Consultas 2009, 11 de marzo]

5. Licencia del Campus de la Universidad de Salamanca.

6. Estos resultados coinciden con las aportaciones de Berrios y Buxarrais, (2005), quienes señalan que desde el punto de vista de los recursos informáticos, las actividades que con mayor frecuencia se realizan son escuchar música, jugar y utilizar el procesador de texto.

7. Las investigaciones al respecto (Moret, 1999; Reading the situation, 2000, entre otros) constatan que la lectura es una actividad de ocio que se incrementa, hasta los 11 años de edad aproximadamente. A partir de ese momento la tendencia va disminuyendo, especialmente entre los varones, de modo que cuando finalizan la escolaridad obligatoria, muchos de ellos manifiestan no tener demasiado interés en leer por placer, considerando esta actividad en muchos casos como algo impuesto desde el ámbito educativo formal.

8. La música en la adolescencia desempeña un importante papel en la socialización, formación de la identidad y autonomía. Los adolescentes emplean de modo natural esta actividad como refuerzo para identificarse con su grupo de iguales, para relajarse, entretenerse, evadirse de la soledad, o incluso como vehículo de rebeldía contra los convencionalismos (Redondo, 2000). 


\section{REFERENCIAS BIBLIOGRÁFICAS}

Arnal, J. et al (1992). Investigación educativa. Fundamentos y metodología. Barcelona: Labor.

Bauman, Z. (2007). Amor líquido. Acerca de la fragilidad de los vínculos humanos. Madrid: Fondo de Cultura Económica.

Berrios, LL. y Buxarrais. $M^{\mathrm{a}}$ R. (2005). Las tecnologías de la información y la comunicación (TIC) y los adolescentes. Algunos datos. Monografías Virtuales. Ciudadanía, Democracia y valores en sociedades plurales. Disponible en http://www.oei.es/valores2/mono grafias/monografia05/reflexion05.htm [2009, 22 de noviembre]

Bransford, J. D., Brown, A. L. y Concking, R. R. (2000). How people learn. Brain, Mind, Experience, and School. Washington, DC: National Academy Press.

Caride, J. A. (1998). Educación del ocio y tiempo libre, en AA. VV. Atención a los espacios y tiempos extraescolares. Granada: Grupo Editorial Universitario, 17-32.

Caride, J. A. (2007). El valor pedagógicosocial del ocio en la sociedad red, en Cid, X. M y Peres, A. (eds.). Educación social, animación sociocultural y desarrollo comunitario. Vigo: Universidade de Vigo, 51-58.

Comas, D. (2000). Agobio y normalidad: una mirada crítica sobre el sector ocio juvenil en la España actual. Revista de estudios de juventud, 50, 9-22.

Cuenca, M. (2004). Pedagogía del ocio: modelos y propuestas. Bilbao: Universidad de Deusto.

Etxeberría, F; y otros (2008). Lectura en la Educación, en Asensio. J. y otros.
Lectura y Educación. Barcelona: Universitat Autónoma de Barcelona, 3-76.

Fernández, A. y Carmona, G. (2009). Rehacer la educación: los mapas del desarrollo humano. Teoría de la Educación. Revista Interuniversitaria, 21(2), 45-78.

Gimeno, J. (2008). El valor del tiempo en educación. Madrid: Morata.

Kerlinger, F., y Lee, H. (2002). Investigación del Comportamiento, Métodos de Investigación en Ciencias Sociales. México: McGraw-Hill.

Hormigos, J. (2010). La creación de identidades culturales a través del sonido. Comunicar, 34/XVII, 91-98.

Lobo, J. L. y Menchén, F. (2004). Libertad y responsabilidad en el tiempo libre. Estrategias y pautas para padres y educadores. Madrid: Pirámide.

Lull, J. (2001). Teoría y práctica de la educación en el tiempo libre. Madrid: CCS.

McNamara, K. J. (1997). Shapes of times. The evolution of growth and development. Baltimore: The Johns Hopkins University Press.

Moret, Z. (1999). Dejad que los libros vengan a mí, o la aventura de leer y escribir creativamente, en Fundación Germán Sánchez Ruipérez Animar a escribir para animar a leer. Salamanca: Fundación Germán Sánchez Ruipérez, 179-90.

Morin, E. (2006). El Método VI: Ética. Madrid: Cátedra.

Muntañola, J. (2007). Las formas del tiempo. Vol. I. Arquitectura, educación y sociedad.Badajoz: @becedario.

Pérez, R. (2005). Calidad de la educación, calidad en la educación. Hacia 
su necesaria integración. Educación XXI, 8, 11-33.

Puig, J. Ma . y Trilla, J. (1987). La Pedagogía del ocio. Barcelona: Laertes.

Reading the Situation (2000). Book reading, buying and borrowing habits in the UK. Book MarketingLtd: The Reading Partnership.

Redondo, A. (2000). El papel de la música en los jóvenes. Disponible en http:77www.comtf.es/pediatría/Congre so_AEP_2000/Ponencias-htm [Consulta: 27-10-2008].

Romero, C. (2000) El conocimiento del tiempo educativo. Barcelona: Alertes.

Romero, C. y otros (2009). Tejiendo vínculos. La textura de la relación educativa, en Peña, J. V. y Férnández, C. M ${ }^{\mathrm{a}}$ (coords.). La escuela en crisis. Barcelona: Octaedro, 105-149.
Santos, J. et al (2004). Diseño de encuestas para estudios de mercado. Técnicas de muestreo y análisis multivariante. Madrid: Centro de Estudios Ramón Areces.

Sarrate, $M^{a}$ L. (2007). Ocio y desarrollo humano, en Cid, X. M. y Peres, A. (eds.). Educación social, animación sociocultural y desarrollo comunitario. Vigo: Universidade de Vigo, 865-874.

Saz-Marín, A. I. (2007). S.O.S. Adolescentes. Madrid: Aguilar.

W.L.R.A. (1993). Carta Internacional de WLRA para la educación del tiempo libre. Disponible en http://www.sportsalut. com.ar/articulos/recreacion/carta\%20i nternacional $\% 20 \mathrm{de} \% 20$ wlra.pdf [Consulta: 2010, 12 de abril]

Zubiri, X. (1996). Espacio. Tiempo. Materia. Madrid: Alianza.

Zuzanek, R. (1980). Work and Leisure. Nueva Cork: Praeger Pub. 


\section{PALABRAS CLAVE}

Tiempo, educación en el tiempo libre, ocio, adolescencia.

\section{KEYWORDS}

Time, leisure education, leisure, adolescence.

\section{PERFIL ACADÉMICO DE LOS AUTORES}

José Manuel Muñoz Rodríguez, Licenciado y Doctor en Pedagogía por la Universidad de Salamanca. Profesor y secretario académico del Departamento de Teoría e Historia de la Educación de la Universidad de Salamanca. Miembro del Grupo de Investigación de Excelencia de la Junta de Castilla y León "Procesos, espacios y prácticas educativas". Sus líneas de investigación se centran en la Teoría de la Educación y, concretamente, en la Pedagogía de los espacios, la educación no formal y la educación ambiental.

Susana Olmos Migueláñez, Doctora en Pedagogía y profesora ayudante de Métodos de Investigación y Diagnóstico en Educación, de la Universidad de Salamanca. Actualmente, ocupa el cargo de secretaria académica del Departamento de Didáctica, Organización y Métodos de Investigación. Miembro de Grupo de Investigación GE2O "Evaluación y Orientación Educativa".

Su línea de investigación se centra en la metodología de investigación y de evaluación, en concreto, en la evaluación en contexto de formación virtual.

Dirección de los autores: Universidad de Salamanca.

Facultad de Educación.

P. ${ }^{\circ}$ de canalejas, 169.

37008 Salamanca.

E-mail: pepema@usal.es

solmos@usal.es

Fecha de Recepción del artículo: 25. Diciembre.2009

Fecha de Revisión del artículo: 15. Febrero. 2010

Fecha de Aceptación del artículo: 1. Junio. 2010 